\title{
Observation of Squeezed States Generated by Four-Wave Mixing in an Optical Cavity
}

\author{
R. E. Slusher \\ AT\&T Bell Laboratories, Murray Hill, New Jersey 07974 \\ L. W. Hollberg \\ AT\&T Bell Laboratories, Holmdel, New Jersey 07733 \\ and \\ B. Yurke, J. C. Mertz, and J. F. Valley ${ }^{(a)}$ \\ AT\&T Bell Laboratories, Murray Hill, New Jersey 07974
}

(Received 27 August 1985)

\begin{abstract}
Squeezed states of the electromagnetic field have been generated by nondegenerate four-wave mixing due to $\mathrm{Na}$ atoms in an optical cavity. The optical noise in the cavity, comprised of primarily vacuum fluctuations and a small component of spontaneous emission from the pumped $\mathrm{Na}$ atoms, is amplified in one quadrature of the optical field and deamplified in the other quadrature. These quadrature components are measured with a balanced homodyne detector. The total noise level in the deamplified quadrature drops below the vacuum noise level.
\end{abstract}

PACS numbers: $42.50 .+\mathrm{q}, 06.30 . \mathrm{Lz}, 42.65 .-\mathrm{k}$

A quantum description of the electromagnetic field includes a broad class of minimum-uncertainty states where the variances in the field quadrature operators $X_{1}$ and $X_{2}$ are limited by

$$
\Delta X_{1} \Delta_{2}=\frac{1}{4} \text {, }
$$

where a particular mode of the electric field is

$$
E=E_{0}\left(X_{1} \cos \omega t+X_{2} \sin \omega t\right),
$$

$X_{1}$ and $X_{2}$ obey the commutator relation

$$
\left[X_{1}, X_{2}\right]=\frac{i}{2} \text {, }
$$

$\omega$ is the frequency, and $E_{0}$ is a constant. Coherent light (e.g., single-mode laser light) belongs to this class and has equal variance for each of the two quadrature components. Squeezed states or squeezed coherent states are fields in the class defined by Eq. (1) but where the quadrature variances are not equal. ${ }^{1,2}$ These squeezed states have a number of unique observable properties which cannot be explained under the assumption of a classical radiation field. For example, as observed in the experiment reported here, the noise level in homodyne detection of these squeezed fields can be reduced below the "shot noise" limit. This shot-noise level is interpreted in semiclassical models as random excitations of the quantized detector electron levels by a classical field. In a fully quantum model this noise arises from vacuum fluctuations. ${ }^{3}$ A reduction in this noise level, as observed in these experiments, is predicted only by the fully quantum model. ${ }^{4}$ An important application of this noise reduction is the interferometric measurement of phase. ${ }^{5}$ It may lead to a major improvement in many precision measurement experiments.
A number of phase-dependent nonlinear optical phenomena have been suggested for generation of squeezed states. ${ }^{1}$ Backward four-wave mixing ${ }^{6}$ near an atomic resonance was chosen for the present experiment because of the relatively large nonlinearity and low optical losses. Generation of squeezed states by four-wave mixing has also been the subject of extensive theoretical study. ${ }^{7-10}$ Several groups have recently reported four-wave-mixing experimental results $^{11-13}$ which approach the generation of squeezed states and demonstrate "squeezing" of noise in the classical limit or near the vacuum level.

In order to enhance the four-wave-mixing gain and restrict the frequency spectrum to a noise-free region, the experiments described here use nondegenerate four-wave mixing in an optical cavity. ${ }^{14}$ The measurement of the squeezed fields is accomplished by matching of the optical cavity mode to the signal port of a balanced homodyne detector. ${ }^{3}$ A schematic diagram of the experimental configuration is shown in Fig. 1. A $\mathrm{cw}$ single-mode ring dye laser pumps a beam of $\mathrm{Na}$ atoms at a frequency which is typically tuned $1.5 \mathrm{GHz}$ above or below (above for the data presented here) the hyperfine components in the $D_{2}$ resonance of $\mathrm{Na}$ at $589.0 \mathrm{~nm}$. The pump is focused in the $1-\mathrm{cm}$-wide $\mathrm{Na}$ beam and enhanced in a confocal buildup cavity with a finesse of 30 . This cavity is servo-controlled to remain at resonance with the pump light. The pump intensity $\left(\sim 100 \mathrm{~W} / \mathrm{cm}^{2}\right)$ is typically near one-tenth the saturation level for the $\mathrm{Na}$ resonance at a detuning of $1 \mathrm{GHz}$. The $\mathrm{Na}$ atomic beam has a density near $10^{11} \mathrm{~cm}^{-3}$ and an angular divergence of $\pm 5^{\circ}$ which results in a Doppler width of $100 \mathrm{MHz}$. The linear absorption coefficient $\alpha_{0}$ is near $5 \mathrm{~cm}^{-1}$ at the center of the weaker hyperfine group. At frequency shifts of 1 


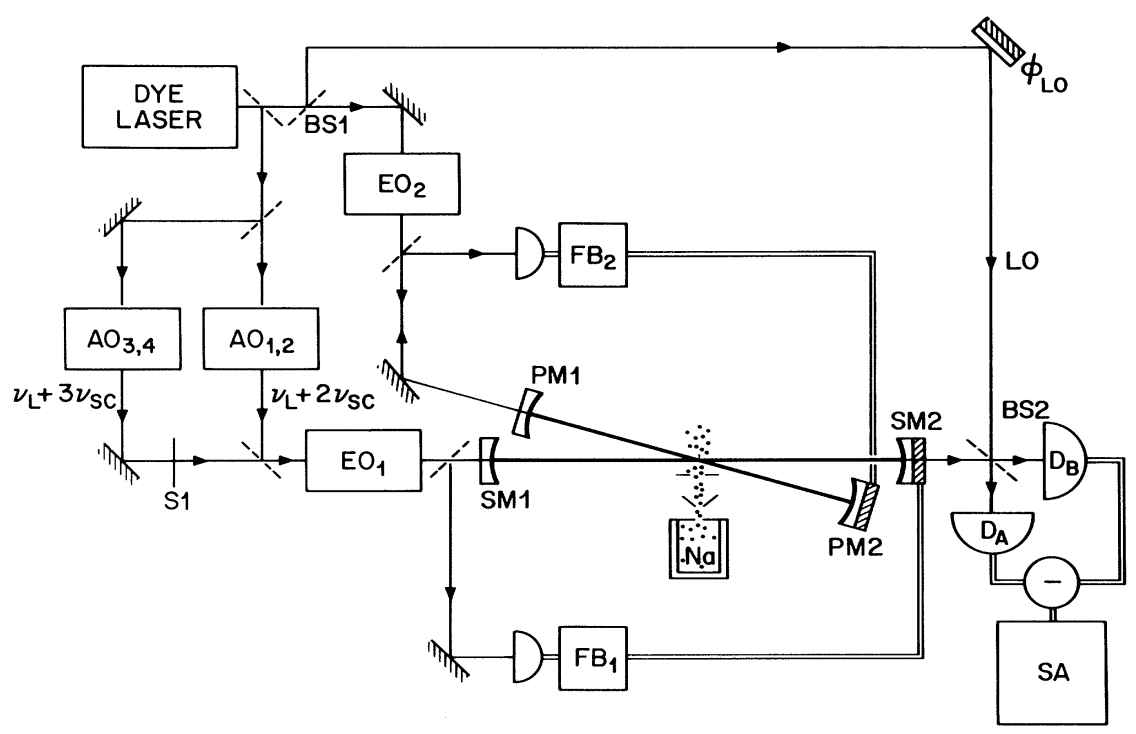

FIG. 1. A schematic diagram of the experimental apparatus for squeezed-state generation by four-wave mixing. A ring dye laser pumps a $\mathrm{Na}$ atomic beam at the pump-cavity (mirrors PM1 and PM2) resonance frequency. The pumped Na atoms generate four-wave-mixing gain in the squeezing cavity (mirrors SM1 and SM2). A local oscillator beam LO is split off by a beam splitter BS1. The squeezed cavity noise is detected with a balanced homodyne detector ( $\operatorname{splitter} \mathrm{BS}_{2}$, detectors $\mathrm{D}_{A}$ and $\mathrm{D}_{B}$ ) and observed on a spectrum analyzer SA. The classical four-wave-mixing gain is measured by opening shutter S1 and injecting into the squeezing cavity a beam which is frequency shifted from the pump by $3 \nu_{\mathrm{sc}}$. This beam is generated by frequency shifters $\mathrm{AO}_{3,4}$. Both pump and squeezed cavities are locked to the dye laser frequency by fm sidebands generated by $\mathrm{EO}_{1}$ and $\mathrm{EO}_{2}$ which are detected to obtain feedbacks $\mathrm{FB}_{1}$ and $\mathrm{FB}_{2}$ to piezoelectric transducers on mirrors PM2 and SM2.

to $2 \mathrm{GHz}$, the linear absorption from this beam is less than $1 \%$ and is limited by the Lorentzian tails of the atomic absorption, not by Doppler broadening.

The squeezed state is generated in a cavity by a linear combination of the conjugate pairs of photons generated by the four-wave-mixing process. This process builds up squeezed fields in the confocal cavity formed by SM1 and SM2 in Fig. 1. The frequencies of the paired photons are symmetrically frequency shifted with respect to the pump frequency. Experimentally, the measured photon pairs are shifted by $\pm 3 \nu_{\mathrm{sc}}= \pm 421.5 \mathrm{MHz}$, where $\nu_{\mathrm{sc}}=140.5 \mathrm{MHz}$ is the cavity mode-spacing frequency. The squeezing cavity is at an angle of $0.86^{\circ}$ with respect to the pump beam and is also servo-controlled to remain resonant at the pump frequency shifted by $2 v_{\mathrm{sc}}=281 \mathrm{MHz}$. Squeezed radiation can be optimally observed outside the squeezing cavity if the mirror reflectivities are not equal. ${ }^{14}$ In this experiment the reflectivities are 0.995 for SM1 and 0.98 for SM2.

Radiation from the 0.98 -reflectivity output mirror is detected by a balanced detector ${ }^{3}$ with an unshifted portion of the pump beam used as the local oscillator (LO). The detectors are $p-i-n$ silicon photodiodes ${ }^{15}$ with quantum efficiencies near 0.7 . Low-noise widebandwidth $^{16}$ amplifiers increase the two photocurrent signals by $60 \mathrm{~dB}$. The noise spectrum of the difference between the two detector signals is observed on a spec- trum analyzer at frequencies near the beat frequency between the LO and squeezed cavity output at frequencies shifted by $\pm 3 \nu_{\text {sc }} \cong 421.5 \mathrm{MHz}$. The balanced detector is carefully aligned to respond only to modes of the squeezed cavity. The homodyne efficiency due to wave-front matching to the cavity mode is typically 0.75 .

In order to understand the squeezing process in detail, it is important to measure the cavity fourwave-mixing power gain in the classical limit. For this purpose, a portion of the pump beam is shifted by $3 \nu_{\mathrm{sc}}$ and coupled into the squeezed cavity by the opening of shutter S1 in Fig. 1. The resulting sharp spectral component is shown in Fig. 2(a) with the pump beam blocked (no four-wave mixing) and with the pump on (four-wave-mixing amplification for one field quadrature and deamplification for the other field quadrature). Here the oscillatory trace is obtained with the pump on and the LO phase swept by a piezoelectrically controlled mirror. An LO phase shift of $\pi / 2$ results in a half-cycle variation of the homodyne detected signal as expected for four-wave mixing. The square of the ratio of the maximum (or minimum) voltage with the pump on to the voltage with the pump blocked is a measure of the classical gain [2.3 for data in Fig. 2(a)].

The observed noise near $421.5 \mathrm{MHz}$ with the pump light blocked is predominantly due to vacuum fluctuations introduced by optical losses and detector-system 


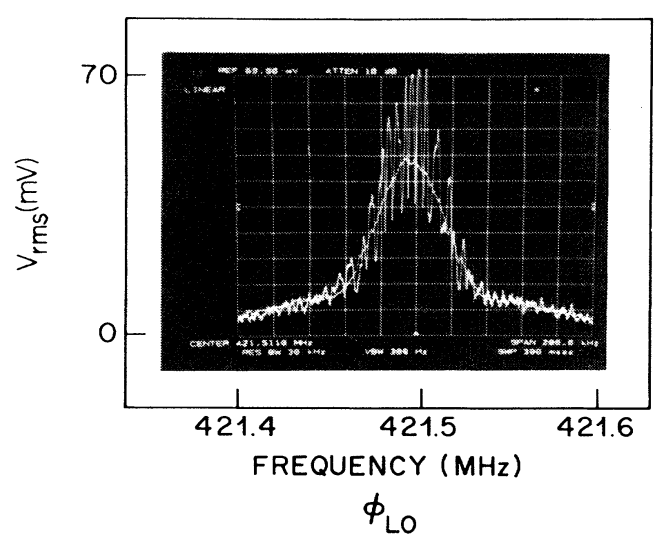

(a)

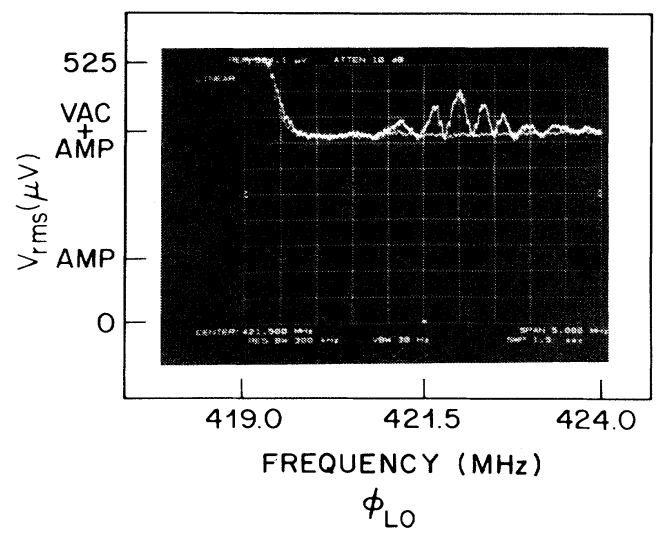

(b)

FIG. 2. (a) A measurement of four-wave-mixing gain. The smooth bell-shaped trace shows the signal resulting from the beam injected via shutter $S 1$ when the pump is off and hence no four-wave-mixing gain is present. The modulated trace is taken with the pump on and is a direct measure of the four-wave-mixing gain. (b) The homodyne detector voltage noise spectra near a pair of cavity resonances spaced at $\pm 3 \nu_{\text {sc }}$ from the pump frequency, with the pump light blocked (dim trace labeled VAC + AMP) and pump light on (bright modulated trace). The amplifier noise level (AMP) is obtained by blocking of the LO beam. Both spectrum analyzer frequency and optical LO phase $( \pm \pi)$ are linearly swept in these pictures. The feature at the extreme left is an extraneous signal due to the LO and some remaining mismatch between the two detectors.

inefficiencies. $^{17}$ A small component of the noise is due to the amplifiers $(\sim 10 \%$ of the total noise power at the spectrum analyzer). This combined vacuum fluctuation and amplifier noise level is shown as the dim horizontal line [labeled "VAC + AMP" in Fig. 2(b)].

The squeezing of the noise in the cavity with the pump light on is shown by the oscillatory trace in Fig. 2(b) as a function of the spectrum analyzer frequency

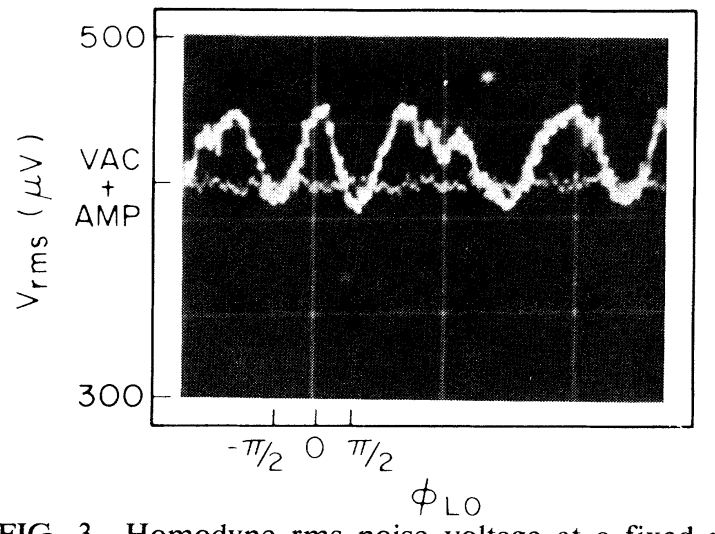

FIG. 3. Homodyne rms noise voltage at a fixed rf frequency as a function of the local oscillator phase with the squeeze cavity blocked (dim horizontal trace) and with the squeeze cavity open to the balanced detector and the pump light on (bright trace). The frequency is fixed at 422.25 $\mathrm{MHz}$ or $0.25 \mathrm{MHz}$ above the frequency where the maximum four-wave-mixing gain is obtained [see Fig. 2(b)]. With the LO on and the cavity and/or pump blocked, the level of the dim trace (labeled VAC + AMP) is quite stable and provides a reliable reference level for detecting the squeezed noise levels. Note that the zero level [similar to that in Fig. 2 (b)] is offset in order to exhibit the noise levels more clearly.

and $\phi_{\text {LO }}$. Four-wave-mixing gain enhanced by the optical cavity is evident over a $2-\mathrm{MHz}$ region centered at $422 \mathrm{MHz}$. This width is approximately the cavity resonance width expected for the mirror reflectivities. The gain is phase dependent as in the classical case [Fig. 2 (a)]. The deamplification below the vacuum level is not as large as expected in the simplest model with no other noise sources. In fact, the noise drops below the vacuum level only near $\pm 0.5-\mathrm{MHz}$ frequency shifts from the cavity resonant frequency. The deamplification below the total noise level is shown in Fig. 3, where the frequency is held constant and $\phi_{\mathrm{LO}}$ is varied. The noise reduction below the vacuum fluctuation (shot noise) level at the maximum deamplification points is $0.3 \mathrm{~dB}$ or nearly $7 \%$ in power (with the small, constant amplifier-noise component taken into account). This deamplification of vacuum fluctuations is an entirely quantum feature of the radiation field and cannot be explained with a semiclassical model. ${ }^{4}$ The phase dependence in Fig. 3 is irregular because of acoustic variations of the LO path. At frequency shifts of more than $3 \mathrm{MHz}$ from the cavity resonance, there is no distinguishable difference between the noise with the cavity or pump blocked and the noise with both cavity unblocked and pump on.

A simple phenomenological model ${ }^{18}$ is used to analyze the data. It includes losses in the cavity and losses in the detection optics which degrade the observed degree of squeezing. This model allows a cal- 
culation of the expected vacuum noise squeezing levels from the measured classical power gain. With the measured values of 0.5 for the effective detection efficiency and $1 \%$ cavity losses, the model predicts a squeezing voltage swing about the vacuum fluctuation level which is twice as large as that observed in Figs. 2 and 3.

Two noise sources have been identified which reduce the observed squeezing below the vacuum level in the present experimental configuration. First, spontaneous-emission radiation is present in the cavity because of absorption and reemission in the Lorentzian tails of the pumped $\mathrm{Na}$ atoms. ${ }^{13,19}$ In this case, the four-wave-mixing gain acts on both the spontaneous emission and the vacuum fluctuations to produce squeezing above and below a level which is the rms sum of these two noise components. For the data in Figs. 2 and 3, the average spontaneous-emission level is approximately $\frac{1}{12}$ photon per mode compared to the vacuum fluctuation level of $\frac{1}{2}$ photon per mode. This contributes to the minimum levels observed by approximately $5 \%$. Second, rapid phase variations $(>20$ $\mathrm{kHz}$ ) in the pump and LO light relative to the squeeze cavity are not corrected by the servo-controlled piezoelectric transducers used at present. This, coupled with the long averaging times $(\sim 0.1 \mathrm{~s})$ used for recording data, results in appreciable increases in the observed minimum squeezed noise levels. Simulations of this phase-jitter noise show that there is a maximum gain above which the noise level will not drop below the vacuum level. This is consistent with the data in Fig. 2, where the noise drops below the vacuum level only for the lower gain at frequencies offset from the cavity resonance.

In summary, the noise level for homodyne detection has been reduced below the vacuum fluctuation (shot noise) level by four-wave mixing in an optical cavity. The measured $7 \%$ noise reduction corresponds to nearly $20 \%$ squeezing of the vacuum fluctuation noise power at the cavity output when system efficiency (0.5) and phase jitter are taken into account. This observation of squeezed states of light confirms longstanding predictions ${ }^{1}$ of this effect based on a quantum description of the electromagnetic field. Much larger squeezing effects should be obtained when the phase jitter noise is brought under control and optical losses are minimized.

We thank L. Corruccini for collaboration in the early phases of this experiment and acknowledge many helpful discussions with S. L. McCall, J. R. Klauder, P. Kumar, and J. H. Shapiro.

(a) Permanent address: Arizona State University, Tempe, Ariz. 85287.
${ }^{1}$ D. F. Walls, Nature (London) 306, 141 (1983), and references therein.

${ }^{2}$ C. M. Caves and B. L. Schumaker, Phys. Rev. A 31, 3068 (1985); B. L. Schumaker and C. M. Caves, Phys. Rev. A 31, 3093 (1985).

${ }^{3}$ H. P. Yuen and V. W. S. Chan, Opt. Lett. 8, 177, 345(E) (1983); B. L. Schumaker, Opt. Lett. 9, 189 (1984).

4J. H. Shapiro, IEEE J. Quantum Electron. 21, 237 (1985).

${ }^{5}$ C. M. Caves, Phys. Rev. D 23, 1693 (1981); R. S. Bondourant and J. H. Shapiro, Phys. Rev. D 30, 2548 (1984); B. Yurke, S. L. McCall, and J. R. Klauder (to be published). ${ }^{6}$ H. P. Yuen and J. H. Shapiro, Opt. Lett. 4, 334 (1979).

${ }^{7}$ M. D. Reid and D. F. Walls, Phys. Rev. A 31, 1622 (1985).

8B. Yurke, Phys. Rev. A 32, 300, 311 (1985).

${ }^{9}$ P. Kumar and J. H. Shapiro, Phys. Rev. A 30, 1568 (1984).

${ }^{10} \mathrm{~J}$. R. Klauder, S. L. McCall, and B. Yurke, to be published.

${ }^{11}$ M. D. Levenson, R. M. Shelby, and S. H. Perlmutter, in Proceedings of the Conference on Lasers and ElectroOptics, Baltimore, Maryland, 21-24 May 1985 (to be published), and in Proceedings of the Seventh International Conference on Laser Spectroscopy, Maui, Hawaii, June 1985, edited by T. W. Haensch and Y. R. Shen, Springer Series in Optical Science Vol. 49 (Springer-Verlag, New York, to be published).

12M. W. Maeda, P. Kumar, and J. H. Shapiro, in Proceedings of the Topical Meeting on Instabilities and Dynamics of Lasers and Nonlinear Optical Systems, Rochester, New York, June 1985 (to be published).

13R. E. Slusher, L. W. Hollberg, B. Yurke, and J. C. Mertz, in Proceedings of the Topical Meeting on Instabilities and Dynamics of Lasers and Nonlinear Optical Systems, Rochester, New York, June 1985 (to be published); R. E. Slusher, B. Yurke, L. W. Hollberg, and J. C. Mertz, in Proceedings of the Seventh International Conference on Laser Spectroscopy, Maui, Hawaii, June 1985, edited by T. W. Haensch and Y. R. Shen, Series in Optical Science Vol. 49 (SpringerVerlag, New York, to be published).

${ }^{14}$ B. Yurke, Phys. Rev. A 29, 408 (1984).

${ }^{15} \mathrm{RCA}$ type 30902E, 800-MHz bandwidth.

${ }^{16}$ Trontech model W500EF, with high-pass filters at 300 $\mathrm{MHz}$ to avoid gain variation due to the locking signal at 281 $\mathrm{MHz}$ (which also exhibits four-wave-mixing gain and attenuation).

${ }^{17}$ The measured rms noise level with only the LO on the detector is equal to the "shot noise" level (or vacuum fluctuation level in the quantum picture) expected for the measured incident power, quantum efficiency, dc photocurrent, and amplifier gain to within $2 \%$ for both detectors. The balance of the detectors typically results in LO common mode rejection of greater than $20 \mathrm{~dB}$.

${ }^{18} \mathrm{R}$. E. Slusher and B. Yurke, in "Frontiers in Quantum Optics" (Hilger, London, to be published).

${ }^{19}$ R. E. Slusher, L. W. Hollberg, B. Yurke, J. C. Mertz, and J. F. Valley, Phys. Rev. A 31, 3512 (1985). 


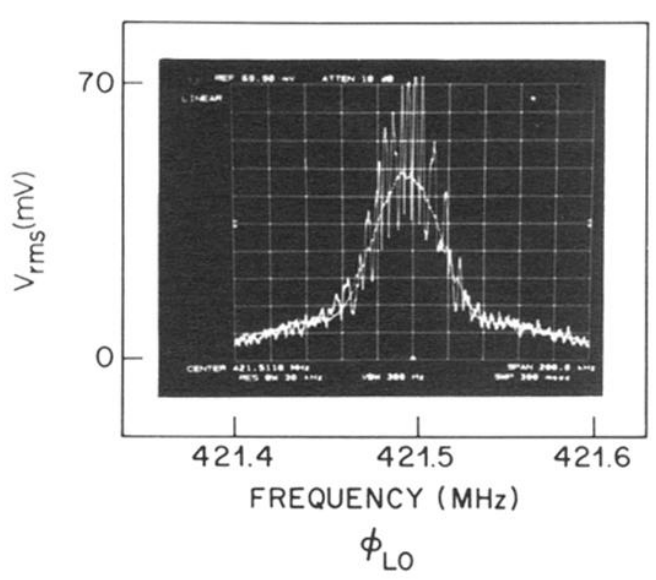

(a)

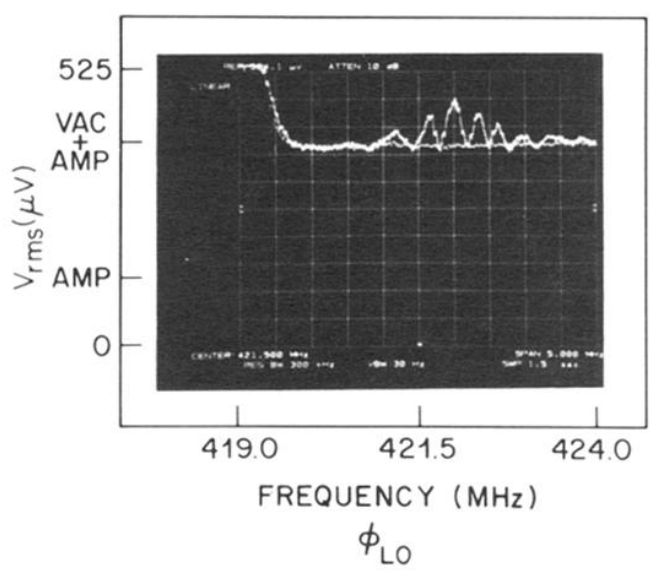

(b)

FIG. 2. (a) A measurement of four-wave-mixing gain. The smooth bell-shaped trace shows the signal resulting from the beam injected via shutter S1 when the pump is off and hence no four-wave-mixing gain is present. The modulated trace is taken with the pump on and is a direct measure of the four-wave-mixing gain. (b) The homodyne detector voltage noise spectra near a pair of cavity resonances spaced at $\pm 3 v_{\mathrm{sc}}$ from the pump frequency, with the pump light blocked (dim trace labeled VAC + AMP) and pump light on (bright modulated trace). The amplifier noise level (AMP) is obtained by blocking of the LO beam. Both spectrum analyzer frequency and optical LO phase $( \pm \pi)$ are linearly swept in these pictures. The feature at the extreme left is an extraneous signal due to the LO and some remaining mismatch between the two detectors. 


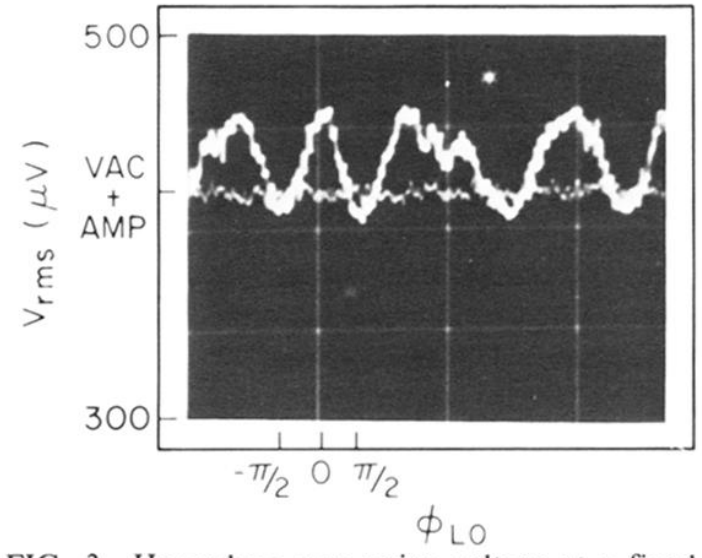

FIG. 3. Homodyne rms noise voltage at a fixed rf frequency as a function of the local oscillator phase with the squeeze cavity blocked (dim horizontal trace) and with the squeeze cavity open to the balanced detector and the pump light on (bright trace). The frequency is fixed at 422.25 $\mathrm{MHz}$ or $0.25 \mathrm{MHz}$ above the frequency where the maximum four-wave-mixing gain is obtained [see Fig. 2(b)]. With the LO on and the cavity and/or pump blocked, the level of the dim trace (labeled VAC + AMP) is quite stable and provides a reliable reference level for detecting the squeezed noise levels. Note that the zero level [similar to that in Fig. 2 (b)] is offset in order to exhibit the noise levels more clearly. 